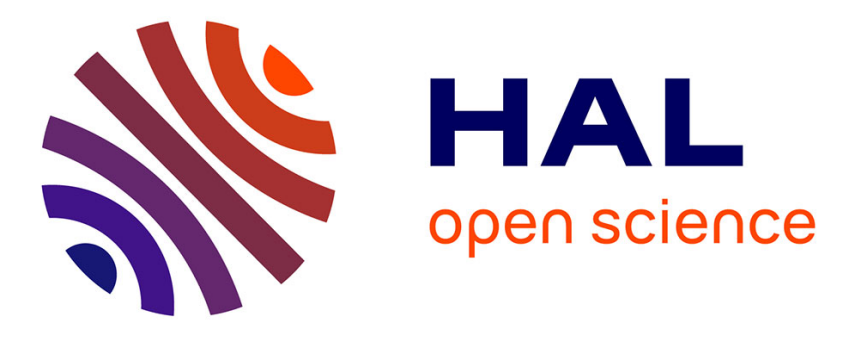

\title{
Algorithms for Adaptive Feedforward Noise Attenuation-A Unified Approach and Experimental Evaluation
}

Tudor-Bogdan Airimitoaie, Ioan Doré Landau, Raul Melendez, Luc Dugard

\section{To cite this version:}

Tudor-Bogdan Airimitoaie, Ioan Doré Landau, Raul Melendez, Luc Dugard. Algorithms for Adaptive Feedforward Noise Attenuation-A Unified Approach and Experimental Evaluation. IEEE Transactions on Control Systems Technology, 2021, 29 (5), pp.1850-1862. 10.1109/TCST.2020.3023276 . hal-02947816

\section{HAL Id: hal-02947816 https://hal.science/hal-02947816}

Submitted on 25 Sep 2020

HAL is a multi-disciplinary open access archive for the deposit and dissemination of scientific research documents, whether they are published or not. The documents may come from teaching and research institutions in France or abroad, or from public or private research centers.
L'archive ouverte pluridisciplinaire HAL, est destinée au dépôt et à la diffusion de documents scientifiques de niveau recherche, publiés ou non, émanant des établissements d'enseignement et de recherche français ou étrangers, des laboratoires publics ou privés. 


\title{
Algorithms for adaptive feedforward noise attenuation - A unified approach and experimental evaluation
}

\author{
Tudor-Bogdan Airimitoaie, Ioan Doré Landau, Raul Melendez, and Luc Dugard
}

\begin{abstract}
Adaptive feedforward broad-band noise compensation is currently used when a correlated measurement with the disturbance (an image of the disturbance) is available. Most of the active feedforward noise control systems feature an internal "positive" acoustical feedback between the compensation system and the disturbance measurement which has to be taken into account. Adaptive algorithms for active feedforward noise attenuation have been developed since 1985 from a local optimization point of view. This paper presents two classes of adaptive configurations for active feedforward noise compensation developed from a stability point of view. The first class uses a standard IIR(FIR) compensator structure while the second class uses the Youla-Kučera parametrization of the feedforward noise compensator. This allows to separate the problem of stabilizing the internal positive feedback loop from the minimization of the residual noise. These adaptive schemes have been developed in the context of active vibration control but, as it will be shown, they can be used also in active noise control. The paper presents in a unified manner the available algorithms and compensator structures for adaptive feedforward noise attenuation and provides a comparative experimental evaluation on a relevant experimental test-bench (a duct silencer).
\end{abstract}

Index Terms-active noise control, adaptive feedforward compensation, Youla-Kučera parametrization, positive feedback coupling.
Manuscript received March 10, 2020; revised May 20, 2020; accepted for publication September 6, 2020. This work was not supported by any grant.

Tudor-Bogdan Airimitoaie is with the Univ. Bordeaux, Bordeaux INP, CNRS, IMS, UMR 5218, 33405 Talence, France (e-mail: tudorbogdan.airimitoaie@ims-bordeaux.fr).

Raul Melendez, Ioan Doré Landau, and Luc Dugard are with the Univ. Grenoble Alpes, CNRS, Grenoble INP, GIPSA-lab, 38000 Grenoble, France (e-mail: raul.melendez, ioan-dore.landau, luc.dugard@gipsalab.grenoble-inp.fr).

\section{LIST OF ACRONYMS}

$\begin{array}{ll}\text { ANC } & \text { Active Noise Control } \\ \text { ANVC } & \text { Active Noise and Vibration Control } \\ \text { AVC } & \text { Active Vibration Control } \\ \text { FIR } & \text { Finite Impulse Response } \\ \text { FULMS } & \text { Filtered-u Least Mean Squares } \\ \text { FUPLR } & \text { Filtered-u Pseudo Linear Regression } \\ \text { FUSBA } & \text { Filtered-u Stability Based Algorithm } \\ \text { IIR } & \text { Infinite Impulse Response } \\ \text { FIRYK } & \text { Youla-Kučera parametrized IIR adaptive } \\ & \text { feedforward compensator using an FIR } \\ & \text { Youla-Kučera filter } \\ \text { IIRYK } & \text { Youla-Kučera parametrized IIR adaptive } \\ & \text { feedforward compensator using an IIR } \\ & \text { Youla-Kučera filter } \\ \text { LMS } & \text { Least Mean Squares } \\ \text { NFULMS Normalized FULMS } \\ \text { PAA } & \text { Parameter Adaptation Algorithm } \\ \text { PRBS } & \text { Pseudo Random Binary Sequence } \\ \text { PSD } & \text { Power Spectral Density } \\ \text { TFUSBA } & \text { Scalar FUSBA } \\ \text { Strictly Positive Real (transfer function) }\end{array}$

\section{INTRODUCTION}

A DAPTIVE feedforward noise attenuation is widely used when a well correlated signal with the disturbance (image of the disturbance) is available ( [1]-[4]). The first references go back roughly to 1985 ( [5]). In most of the systems, there is a positive acoustic coupling between the feedforward compensation system and the measurement of the image of the disturbance. This often leads to the instability of the system. In the context of this inherent "positive" feedback, the adaptive feedforward compensator should minimize the effect of the disturbance while simultaneously assuring the stability of the internal positive feedback loop. This problem has been clearly identified by the mid nineties [6], [7]. 


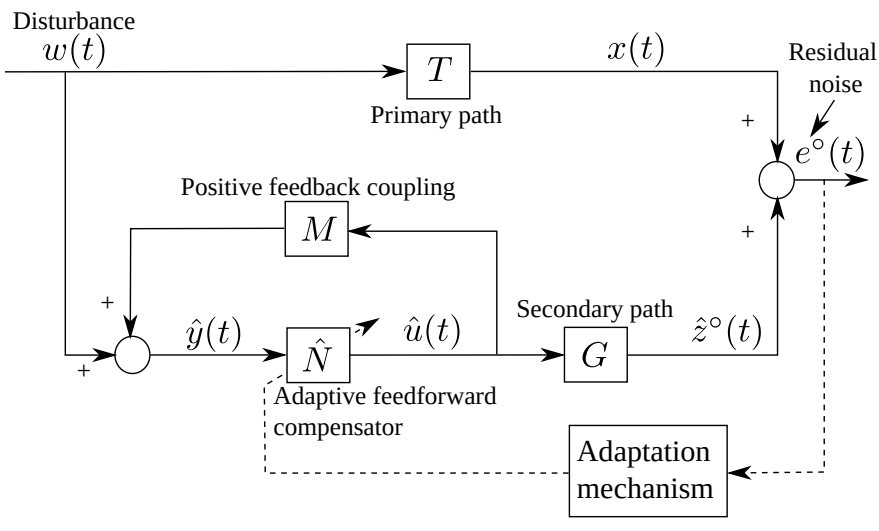

Fig. 1. Feedforward AVC with adaptive feedforward compensator.

Figure 1 gives the basic block diagram of the adaptive feedforward compensation in the presence of the internal positive coupling between the output of the compensator and the measurement of the image of the incoming noise. The incoming noise propagates through the so called primary path $T$ and its effect is compensated by a secondary noise source through the secondary path $G$ driven by a feedforward compensator $\hat{N}$. The input to the feedforward compensator is the sum of the image of the incoming noise and of the internal acoustical positive feedback through $M$. The residual noise is used to emulate the adaptation of the feedforward compensator.

At the end of the nineties, adaptive feedback noise control emerged as an efficient solution for cancelling single or multiple tonal disturbances ( [8]), ( [9]) taking advantage of the internal model principle and the Youla-Kučera parametrization of the feedback controller. Nevertheless, the efficient use of the feedback approach for attenuation of broad-band noise is limited by the Bode integral. Therefore one can say that the adaptive feedforward noise compensation is particularly dedicated to the attenuation of broad-band noise with unknown and time varying characteristics. For this reason the present paper will focus on the experimental evaluation of the various feedforward compensator structures and adaptation algorithms in the presence of broad-band noise disturbances.

A major component of such a system is the PAA. In the field of ANC, the first algorithm used was the so called LMS ( [5]) derived from a local minimization of a quadratic criterion in terms of the residual noise. Many contributions have been done on the analysis of the properties of this algorithm ${ }^{1}$ and the improvement of the algorithm. Filtering of the regressor vector was one of the ways for improving the adaptation algorithm and the FULMS algorithm $([10]-[12])^{2}$ seems to be the most used algorithm in recent publications ( [13], [14]).

In ANC, a first reference for a stability approach in the presence of the internal positive feedback is ( [7]). Unfortunately, the applicability of the results is very limited since

\footnotetext{
${ }^{1}$ However these attempts have not solved the stability problem in the presence of the internal "positive" feedback

${ }^{2}$ It is used with an IIR structure of the feedforward compensator. When it is used with a FIR feedforward compensator it is denoted FXLMS.
}

one assumes that the secondary (compensatory) path has a simple positive gain or it is characterized by a strictly positive real transfer function (unrealistic hypothesis). In the field of AVC the paper [15] provides a full synthesis procedure for asymptotically stable adaptation algorithms using IIR feedforard compensators in the presence of the internal feedback. These algorithms can be used also in ANC as it will be shown in this paper. It is important to note that the FULMS algorithm can be viewed as a particular approximation of the algorithms derived from stability considerations in [15].

An interesting idea is presented in the paper [4]: separate the stabilization of the internal positive feedback loop from the minimization of the residual noise. This can be done by using a Youla-Kučera parametrization of the feedforward compensator. A tuning procedure based on system identification has been proposed and tested on a noise silencer. This idea has been used in [16], [17] for developing direct adaptive feedforward compensation schemes using YoulaKučera parametrization of FIR or IIR form for the feedforward compensator. These algorithms have been extensively tested and compared with other algorithms in the field of AVC [17], [18]. Nevertheless, they can be used also in the field of ANC as it will be shown in this paper. They have two advantages with respect to IIR feedforward compensators:

- Possibility to pre-assign the poles of the internal positive closed-loop (not possible with IIR (FIR) feedforward compensators);

- Easier satisfaction of the positive real conditions for stability.

The objectives of this paper are:

- To present in a unified manner the various compensator structures and stability based PAA used in ANVC;

- To comparatively evaluate experimentally in the context of ANC the various algorithms developed in AVC from the stability point of view as well as the algorithms currently used in ANC for attenuating broad-band noise disturbances.

The experimental evaluation of the various algorithms and compensator configurations is done under identical protocols on an experimental test-bench which represents the core of a duct silencer.

The paper is organized as follows: in Section II, the experimental setting is presented. A unified presentation of all the structures and adaption algorithms is given in Section III. In Section IV the general results are particularized for the various compensator configurations. Section V summarizes the experimental results obtained with various structures of the compensator and various parameter adaptation algorithms. Conclusions of these evaluations are given in Section VI.

\section{EXPERIMENTAL Setup}

The view of the test-bench used for experiments is shown in Fig. 2 and its detailed scheme is given in Fig. 3. The actual dimensions of the test-bench are given in Fig. 4.

The speaker used as the source of disturbances is labelled as 1, while the control speaker is marked as 2. At pipe's open end, the microphone that measures the system's output 


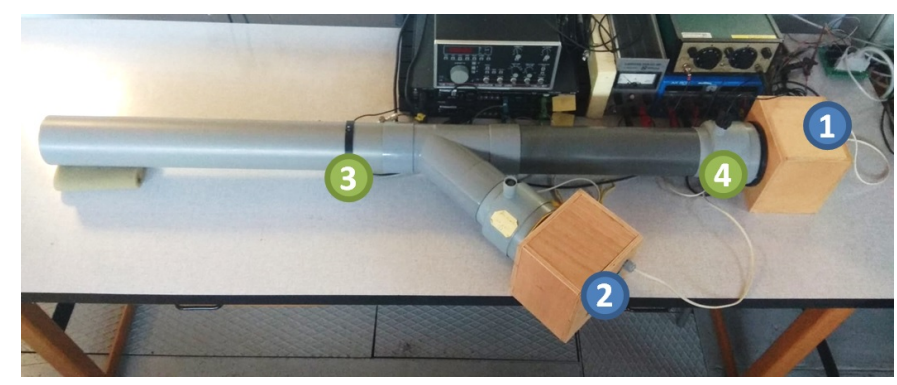

Fig. 2. Duct active noise control test-bench (Photo).

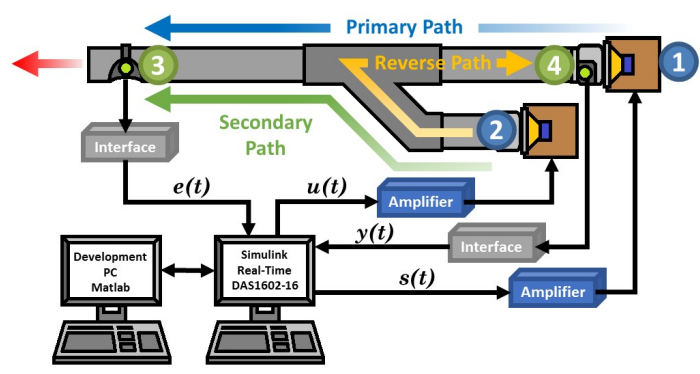

Fig. 3. Duct active noise control test-bench diagram.

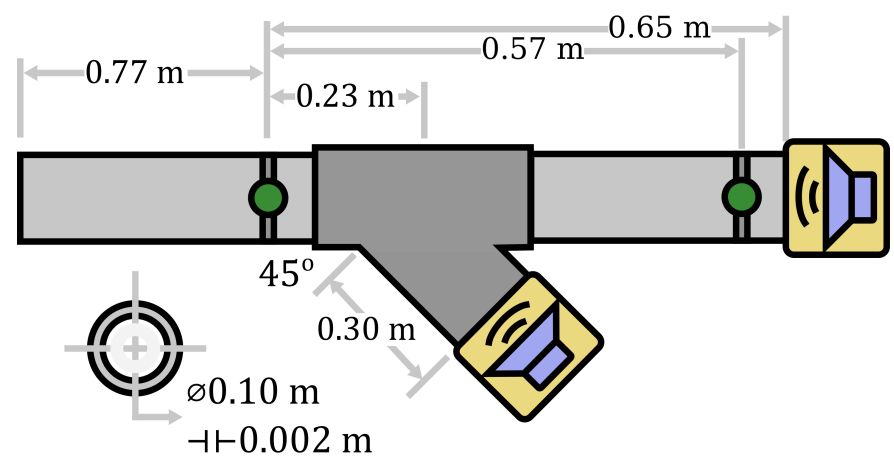

Fig. 4. Duct active noise control test-bench dimensions.

(residual noise $e(t)$ ) is denoted as 3 . Inside the pipe, close to the source of disturbances, the second microphone, labelled as 4 , measures the perturbations' image, denoted as $y(t)$. Additionally, we denote $u(t)$ the control signal, and $s(t)$ the disturbance. The transfer function between the disturbance's speaker and the microphone $(1 \rightarrow 3)$ is called Global Primary Path, while the transfer function between the control speaker and the microphone $(2 \rightarrow 3)$ is denoted Secondary Path. The transfer function between microphones $(4 \rightarrow 3)$ is called Primary Path. The internal coupling found between $(2 \rightarrow 4)$ is denoted Reverse Path. These marked paths have a double differentiator behaviour, since as input we have the voice coil displacement and as output the air acoustic pressure. The speakers are isolated inside wood boxes filled with special foam in order to create anechoic chambers and reduce the radiation noise produced.

Both speakers are connected to a xPC Target computer with Simulink Real-time ${ }^{\circledR}$ environment through a pair of high definition power amplifiers and a data acquisition board. A second computer is used for development, design and operation with Matlab ${ }^{\circledR}$. The sampling frequency has been chosen in accordance with the recommendations given in [18]. Taking into account that disturbances up to $400 \mathrm{~Hz}$ may need to be attenuated, a sampling frequency $f_{s}=2500 \mathrm{~Hz}$ has been chosen $\left(T_{s}=0.0004 \mathrm{sec}\right)$, i.e., approximately six times the maximum frequency to attenuate.

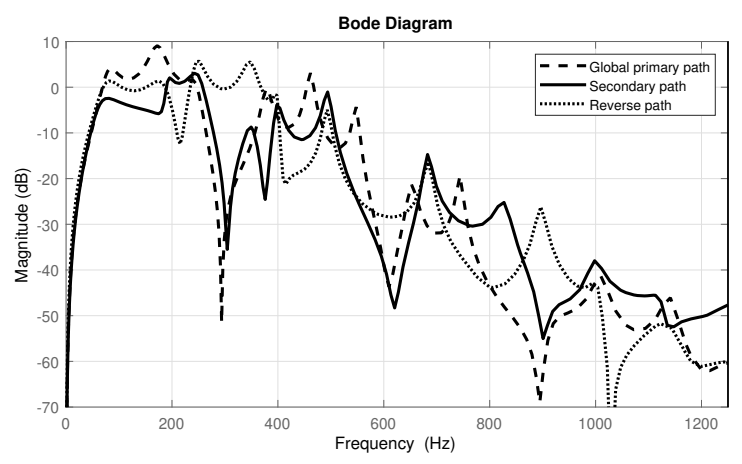

Fig. 5. Frequency characteristics of the Primary, Secondary and Reverse paths identified models.

The frequency characteristics of the identified models for the primary ${ }^{3}$, secondary and reverse paths are shown in Fig. 5. These characteristics present multiple resonances (low damped complex poles) ${ }^{4}$ and anti-resonances (low damped complex zeros). One can see that the secondary path has a high gain between 70 to $270 \mathrm{~Hz}$ which means that disturbances can be efficiently attenuated in this zone. It is also clear that the reverse path has a significant gain on a large frequency range (up to $400 \mathrm{~Hz}$ ) so its effect can not be neglected.

It is important to note that most of the implementations of the adaptive feedforward compensation systems are close to a co-location of the residual noise measurement and of the secondary source used for compensation. See for example [4], [19], [20]. A ratio of $1 / 6$ to $2 / 6$ between the length of the secondary path and the length of the primary path is used in these references. Nevertheless, there are new potential applications areas (exhaust noise reduction on boats, trucks, cars) where thermal constraints will not allow to have a configuration close to a co-location. For this reason the ratio between the length of secondary path and the length of the primary path has been chosen about 5/6 (close to the theoretical limit - see next section).

\section{BASIC EqUATIONS AND NOTATIONS}

The block diagram associated with various configurations of the adaptive feedforward compensators can be described as particular cases of a generalized Youla-Kučera structure for adaptive feedforward compensators shown in Fig. 6.

The primary $(T)$, secondary $(G)$, and reverse (positive coupling) $(M)$ paths represented in Fig. 6 are characterized

\footnotetext{
${ }^{3}$ The primary path model has been exclusively used for simulation purposes.

${ }^{4}$ The lowest damping is around 0.01 .
} 


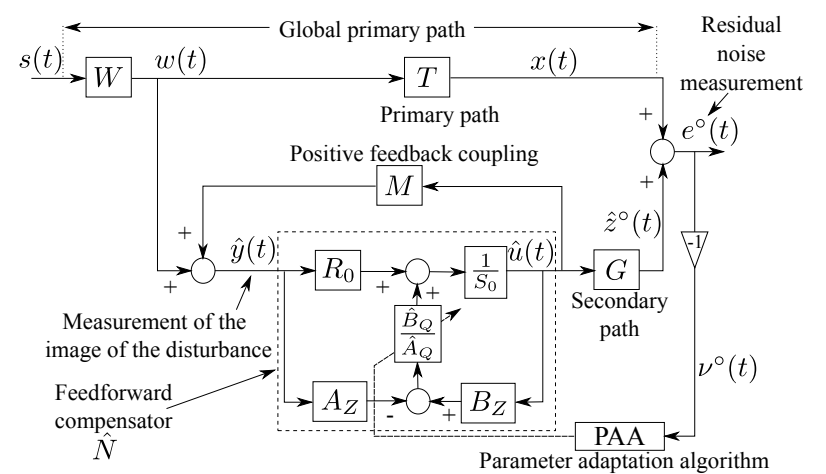

Fig. 6. Adaptive feedforward disturbance compensation using the generalized Youla-Kučera parametrization.

by the asymptotically stable transfer operators:

$$
\begin{aligned}
X\left(q^{-1}\right) & =\frac{B_{X}}{A_{X}}=\frac{q^{-1} B_{X}^{*}\left(q^{-1}\right)}{A_{X}\left(q^{-1}\right)} \\
& =\frac{q^{-d_{X}}\left(b_{1}^{X} q^{-1}+\ldots+b_{n_{B_{X}}}^{X} q^{-n_{B_{X}}}\right)}{1+a_{1}^{X} q^{-1}+\ldots+a_{n_{A_{X}}}^{X} q^{-n_{A_{X}}}},
\end{aligned}
$$

for any $X \in\{T, G, M\} . \hat{G}=\frac{\hat{B}_{G}}{\hat{A}_{G}}, \hat{M}=\frac{\hat{B}_{M}}{\hat{A}_{M}}$, and $\hat{T}=\frac{\hat{B}_{T}}{\hat{A}_{T}}$ denote the identified (estimated) models of $\mathrm{G}, \mathrm{M}$, and $\mathrm{T}$.

Polynomials $A_{Z}$ and $B_{Z}$ are defined as:

$$
\begin{gathered}
A_{Z}=a_{0}^{Z}+a_{1}^{Z} q^{-1}+\ldots \\
B_{Z}=b_{1}^{Z} q^{-1}+\ldots
\end{gathered}
$$

The optimal feedforward compensator which will minimize the residual noise can be written as:

$$
N=\frac{R}{S}=\frac{A_{Q} R_{0}-B_{Q} A_{Z}}{A_{Q} S_{0}-B_{Q} B_{Z}}
$$

where the optimal filter $Q\left(q^{-1}\right)$ has an IIR structure

$$
Q=\frac{B_{Q}}{A_{Q}}=\frac{b_{0}^{Q}+b_{1}^{Q} q^{-1}+\ldots+b_{n_{B_{Q}}}^{Q} q^{-n_{B_{Q}}}}{1+a_{1}^{Q} q^{-1}+\ldots+a_{n_{A_{Q}}}^{Q} q^{-n_{A_{Q}}}}
$$

and $R_{0}\left(q^{-1}\right), S_{0}\left(q^{-1}\right)=1+q^{-1} S_{0}^{*}\left(q^{-1}\right)$ are the polynomials of the central (stabilizing) filter and $A_{Z}\left(q^{-1}\right), B_{Z}\left(q^{-1}\right)$ are given in $(3)^{5}$ and $(4)^{6}$.

The estimated $Q$ IIR filter is denoted by $\hat{Q}\left(q^{-1}\right)$ or $\hat{Q}\left(\hat{\theta}, q^{-1}\right)$ when it is a linear filter with constant coefficients or $\hat{Q}\left(t, q^{-1}\right)$ during estimation (adaptation). The vector of parameters of the optimal QIIR filter will be denoted by

$$
\theta^{T}=\left[b_{0}^{Q}, \ldots, b_{n_{B_{Q}}}^{Q}, a_{1}^{Q}, \ldots, a_{n_{A_{Q}}}^{Q}\right]=\left[\theta_{B_{Q}}^{T}, \theta_{A_{Q}}^{T}\right] .
$$

The vector of parameters for the estimated $\hat{Q} I I R$ filter

$$
\hat{Q}\left(q^{-1}\right)=\frac{\hat{B}_{Q}\left(q^{-1}\right)}{\hat{A}_{Q}\left(q^{-1}\right)}=\frac{\hat{b}_{0}^{Q}+\hat{b}_{1}^{Q} q^{-1}+\ldots+\hat{b}_{n_{B_{Q}}^{Q}} q^{-n_{B_{Q}}}}{1+\hat{a}_{1}^{Q} q^{-1}+\ldots+\hat{a}_{n_{A_{Q}}}^{Q} q^{-n_{A_{Q}}}}
$$

\footnotetext{
${ }^{5}$ The following notation for polynomials will be used throughout this paper: $A\left(q^{-1}\right)=a_{0}+\sum_{i=1}^{n_{A}} a_{i} q^{-i}=a_{0}+q^{-1} A^{*}\left(q^{-1}\right)$.

${ }^{6}$ For $A_{Z}=A_{M}, B_{Z}=B_{M}$ one has the standard YK parametrization used in [17].
}

is denoted by

$$
\hat{\theta}^{T}=\left[\hat{b}_{0}^{Q}, \ldots, \hat{b}_{n_{B_{Q}}}^{Q}, \hat{a}_{1}^{Q}, \ldots, \hat{a}_{n_{A_{Q}}^{Q}}^{Q}\right]=\left[\hat{\theta}_{B_{Q}}^{T}, \hat{\theta}_{A_{Q}}^{T}\right] .
$$

The input of the feedforward filter (called also reference) is denoted by $\hat{y}(t)$ and it corresponds to the measurement provided by the primary microphone. In the absence of the compensation loop (open-loop operation) $\hat{y}(t)=w(t)$ ). The output of the feedforward compensator (which is the control signal applied to the secondary path) is denoted by $\hat{u}(t+1)=$ $\hat{u}(t+1 / \hat{\theta}(t+1))$ (a posteriori output). The a posteriori output of the estimated feedforward compensator using an IIRYK parametrization for the case of time-varying parameter estimates is given by (using (5)):

$$
\begin{aligned}
\hat{u}(t+1)= & \hat{u}(t+1 / \hat{\theta}(t+1)) \\
= & -\hat{S}^{*}\left(t+1, q^{-1}\right) \hat{u}(t)+\hat{R}\left(t+1, q^{-1}\right) \hat{y}(t+1) \\
= & -S_{0}^{*} \hat{u}(t)+R_{0} \hat{y}(t+1)-\hat{A}_{Q}\left(t+1, q^{-1}\right)^{*} \beta(t) \\
& +\hat{B}_{Q}\left(t+1, q^{-1}\right) \alpha(t+1),
\end{aligned}
$$

where (see also Fig. 6).

$$
\begin{aligned}
\alpha(t+1) & =B_{Z} \hat{u}(t+1)-A_{Z} \hat{y}(t+1)= \\
& =B_{Z}^{*} \hat{u}(t)-A_{Z} \hat{y}(t+1) \\
\beta(t) & =S_{0} \hat{u}(t)-R_{0} \hat{y}(t) .
\end{aligned}
$$

In Fig. $6, \nu^{\circ}(t)$ represents the measured a priori adaptation error (it depends upon $\hat{\theta}(t-1)$ ). The a posteriori adaptation error will be denoted by $\nu(t)$ (it depends upon $\hat{\theta}(t))^{7}$. The objective is to develop stable recursive algorithms for the adaptation of the $\mathrm{Q}$ filter parameters such that the measured residual error (noise in ANC) be minimized in the sense of a certain criterion. This has to be done for broad-band disturbances $w(t)$ (or $s(t)$ ) with unknown and variable spectral characteristics and an unknown primary path model.

The algorithms for adaptive feedforward compensation have been developed under the following basic hypotheses:

H1. (Perfect matching condition) There exists an optimal value of the $\mathrm{Q}$ parameters such that

$$
\frac{G \cdot A_{M}\left(R_{0} A_{Q}-A_{Z} B_{Q}\right)}{A_{Q}\left(A_{M} S_{0}-B_{M} R_{0}\right)-B_{Q}\left(B_{Z} A_{M}-B_{M} A_{Z}\right)}=-T .
$$

$\mathrm{H} 2$. The characteristic polynomial of the internal closed-loop for $A_{Q}=1$ and $B_{Q}=0$

$$
P_{0}\left(z^{-1}\right)=A_{M}\left(z^{-1}\right) S_{0}\left(z^{-1}\right)-B_{M}\left(z^{-1}\right) R_{0}\left(z^{-1}\right)
$$

is a Hurwitz polynomial.

H3. (Stability of the internal loop) The characteristic polynomial of the internal closed-loop for the values of $A_{Q}$ and $B_{Q}$ assuring perfect matching is a Hurwitz polynomial:

$$
P=A_{Q}\left(A_{M} S_{0}-B_{M} R_{0}\right)-B_{Q}\left(B_{Z} A_{M}-B_{M} A_{Z}\right)
$$

A first step in the development of the algorithms is to establish for a fixed estimated compensator a relation between the error on the Q-parameters (with respect to the optimal values) and the adaptation error $\nu$. This is summarized in the following lemma.

\footnotetext{
${ }^{7}$ For constant estimated parameters $\nu^{\circ}(t)=\nu(t)$.
} 
Lemma 3.1: Under the hypotheses $\mathrm{H} 1-\mathrm{H} 3$ for the system described by (1) through (11b) using an estimated generalized Youla-Kučera parameterized feedforward compensator with constant parameters, one has:

$$
\nu(t+1 / \hat{\theta})=\frac{A_{M} G}{A_{Q} P_{0}-B_{Q}\left(B_{Z} A_{M}-B_{M} A_{Z}\right)}[\theta-\hat{\theta}]^{T} \phi(t),
$$

with $\phi(t)$ given by:

$$
\begin{aligned}
\phi^{T}(t)=\left[\alpha(t+1), \alpha(t), \ldots, \alpha\left(t-n_{B_{Q}}+1\right),\right. & \\
& \left.-\beta(t),-\beta(t-1), \ldots,-\beta\left(t-n_{A_{Q}}\right)\right] .
\end{aligned}
$$

The proof of this lemma follows the proof given in Appendix A of [17] with the appropriate change of notations and is omitted.

As will be shown subsequently, for assuring the stability of the system, one needs to filter the observation vector $\phi(t)$. Filtering the vector $\phi(t)$ through an asymptotically stable filter $L\left(q^{-1}\right)=\frac{B_{L}}{A_{L}}$, (12) for $\hat{\theta}=$ constant becomes

$$
\begin{array}{r}
\nu(t+1 / \hat{\theta})=\frac{A_{M} G}{\left(A_{Q} P_{0}-B_{Q}\left(B_{Z} A_{M}-B_{M} A_{Z}\right)\right) L} . \\
\cdot[\theta-\hat{\theta}]^{T} \phi_{f}(t)
\end{array}
$$

with

$$
\begin{array}{r}
\phi_{f}(t)=L\left(q^{-1}\right) \phi(t)=\left[\alpha_{f}(t+1), \ldots, \alpha_{f}\left(t-n_{B_{Q}}+1\right),\right. \\
\left.\beta_{f}(t), \beta_{f}(t-1), \ldots, \beta_{f}\left(t-n_{A_{Q}}\right)\right]
\end{array}
$$

where

$$
\alpha_{f}(t+1)=L\left(q^{-1}\right) \alpha(t+1), \beta_{f}(t)=L\left(q^{-1}\right) \beta(t) .
$$

When the parameters of $\hat{Q}$ evolve over time and neglecting the non-commutativity of the time-varying operators, (14) transforms into ${ }^{8}$

$$
\begin{aligned}
\nu(t+1 / \hat{\theta}(t+1))=\frac{A_{M} G}{\left[A_{Q} P_{0}-B_{Q}\left(B_{Z} A_{M}-B_{M} A_{Z}\right)\right] L} . \\
\cdot[\theta-\hat{\theta}(t+1)]^{T} \phi_{f}(t) .
\end{aligned}
$$

\section{Parameter Adaptation Algorithms}

Equation (17) has the standard form for an a posteriori adaptation error ( [21]), which immediately suggests to use the following parameter adaptation algorithm (PAA):

$$
\begin{aligned}
\hat{\theta}(t+1) & =\hat{\theta}(t)+F(t) \psi(t) \nu(t+1) \\
\nu(t+1) & =\frac{\nu^{\circ}(t+1)}{1+\psi^{T}(t) F(t) \psi(t)} ; \\
F(t+1) & =\frac{1}{\lambda_{1}(t)}\left[F(t)-\frac{F(t) \psi(t) \psi^{T}(t) F(t)}{\frac{\lambda_{1}(t)}{\lambda_{2}(t)}+\psi^{T}(t) F(t) \psi(t)}\right] \\
1 & \geq \lambda_{1}(t)>0 ; 0 \leq \lambda_{2}(t)<2 ; F(0)>0 \\
\psi(t) & =\phi_{f}(t)
\end{aligned}
$$

where $\lambda_{1}(t)$ and $\lambda_{2}(t)$ allow to obtain various time profiles for the matrix adaptation gain $F(t)$ (see [21]). By taking $\lambda_{2}(t) \equiv$ 0 and $\lambda_{1}(t) \equiv 1$, one gets a constant adaptation gain matrix.

\footnotetext{
${ }^{8}$ Nevertheless, exact algorithms can be developed taking into account the non-commutativity of the time varying operators - see [21].
}

In the context of this paper we will be interested by two types of adaptation gain allowing to operate in an "adaptive" regime.

- Constant trace algorithm. $\lambda_{1}(t)$ and $\lambda_{2}(t)$ are adjusted continuously to maintain constant the trace of the adaptation gain matrix. This allows to move in the optimal direction while maintaining the adaptation capabilities.

- Constant scalar adaptation gain. This is obtained by taking $\lambda_{1}(t) \equiv 1, \lambda_{2}(t) \equiv 0$ and $F(t)=\gamma I, \gamma>0$ where $\mathrm{I}$ is the identity matrix.

The values of $\lambda_{1}(t)$ and $\lambda_{2}(t)$ in order to maintain constant the trace of the adaptation gain matrix are determined from the equation:

$$
\operatorname{tr}(F(t+1))=\frac{1}{\lambda_{1}(t)} \operatorname{tr}\left(F(t)-\frac{F(t) \psi(t) \psi^{T}(t) F(t)}{\delta(t)+\psi^{T}(t) F(t) \psi(t)}\right)
$$

fixing the ratio $\delta(t)=\lambda_{1}(t) / \lambda_{2}(t)=$ const. Typical value: $\delta=1$.

The updating of matrix $\mathrm{F}(\mathrm{t})$ is done using the U-D factorization for numerical robustness reasons. The details of this algorithm ${ }^{9}$ are given in [18, Appendix B].

By taking $F(t)=\gamma I$, where I is the identity matrix, one gets a scalar adaptation gain. The equation (18) for updating the parameter vector becomes:

$$
\hat{\theta}(t+1)=\hat{\theta}(t)+\gamma \psi(t) \frac{\nu^{\circ}(t+1)}{1+\gamma \psi^{T}(t) \psi(t)} .
$$

When using scalar adaptation gain, for very small values of $\gamma$ one can approximate the above equation by

$$
\hat{\theta}(t+1)=\hat{\theta}(t)+\gamma \psi(t) \nu^{\circ}(t+1)
$$

In the FULMS and FXLMS algorithms, since the adaptation gain is small and therefore the residual error will vary slowly, the quantity $\psi(t) \nu^{\circ}(t+1)$ is approximated by $\psi(t-1) \nu^{\circ}(t)$ leading to:

$$
\hat{\theta}(t+1)=\hat{\theta}(t)+\gamma \psi(t-1) \nu^{\circ}(t)
$$

\section{Stability conditions}

Taking into account (17) for the a posteriori adaptation error and the equations for the PAA (18) through (22) one has the following condition for global asymptotic stability [21]:

$$
H^{\prime}\left(z^{-1}\right)=H\left(z^{-1}\right)-\frac{\lambda_{2}}{2}, \quad \max _{t}\left(\lambda_{2}(t)\right) \leq \lambda_{2}<2
$$

with

$$
H\left(z^{-1}\right)=\frac{A_{M} G}{\left(A_{Q} P_{0}-B_{Q}\left(B_{Z} A_{M}-B_{M} A_{Z}\right)\right) L}
$$

should be a strictly positive real (SPR) transfer function. For constant adaptation gains $\left(\lambda_{2}(t)=0\right)$ the stability condition becomes: $H\left(z^{-1}\right)$ should be SPR. ${ }^{10}$

\footnotetext{
${ }^{9}$ Routines for the implementation of the algorithm can be downloaded from http://www.gipsa-lab.grenoble-inp.fr/ ioandore. landau/adaptivecontrol/

${ }^{10}$ The SPR condition can be also interpreted as a condition that the angle between the direction of adaptation and the direction of the inverse of the true gradient (not computable) should be less than $90^{\circ}$. See also [15].
} 


\section{The Filter $L$}

A key role in the stability of the various adaptation algorithms is played by the filter $L$ operating on the observation vector $\phi$. It helps to satisfy the "strictly positive real condition" for asymptotic stability.

Two choices for the filter $L$ will be considered, leading to different algorithms. For the case of matrix adaptation gain one has:

FUPLR: $L=\hat{G}$

$$
\text { FUSBA }: L=\frac{\hat{A}_{M} \hat{G}}{\left[\hat{A}_{Q} \hat{P}_{0}-\hat{B}_{Q}\left(B_{Z} \hat{A}_{M}-\hat{B}_{M} A_{Z}\right)\right]}
$$

The algorithm FUPLR, assuming that the SPR condition given in Eq. (26) is satisfied, assures a global stability of the algorithm for any initial conditions. The SPR stability condition can be relaxed for low adaptation gain provided that in the average the SPR condition is true (see [15], [22], [23]) but the performance will be impacted. To improve the performance one has to use the FUSBA algorithm which tries to make the $H\left(z^{-1}\right)$ transfer function close to 1 . The implementation of the FUSBA algorithm requires real-time estimates of $\hat{A}_{Q}$ and $\hat{B}_{Q}$. To obtain a first estimation of $\hat{A}_{Q}$ and $\hat{B}_{Q}$ one uses the FUPLR algorithm over a certain horizon. Therefore in order to use this algorithm an initialization with the FUPLR algorithm should be done (except for YKFIR). It assumes indeed that the estimated filter $\mathrm{L}$ is asymptotically stable. This requires inclusion of a stability test. The FUPLR stability condition is "global" while the FUSBA condition is "local"11 (except for YKFIR where it is global).

For the constant scalar adaptation gain one has the same choices for the filter $L$ and the corresponding algorithms issued from stability considerations are NFULMS and SFUSBA. Same considerations as for the matrix adaptation gain are valid in the case of constant scalar adaptation gain. The SFUSBA should be initialized using the NFULMS. Note also that FULMS and NFULMS use the same type of filter.

\section{Filtering of the residual error}

An interesting practical issue is the use of a filtered residual error (noise) in the adaptation algorithm. This idea comes from adaptive filtering and identification ( [7], [24]). For a general presentation see [18]. On one hand the use of this filtering may contribute to satisfy the SPR condition for stability and on the other hand (which is the most important) it will shape the resulting spectral density. ${ }^{12}$

In this case, the adaptation error takes the form

$$
\nu^{\circ}(t)=-\left[e^{\circ}(t)+V^{*}\left(q^{-1}\right) e(t-1)\right],
$$

where the filter $V\left(q^{-1}\right)$ is given as

$$
V\left(q^{-1}\right)=1+v_{1} q^{-1}+\ldots+v_{n_{V}} q^{-n_{V}}=1+q^{-1} V^{*}\left(q^{-1}\right) .
$$

\section{Specific Compensator Structures}

Table I summarizes the various particular cases of the compensator structures and gives the corresponding references.

\footnotetext{
${ }^{11}$ Strictly speaking, it is valid only in the neighborhood of the equilibrium point.

${ }^{12}$ In fact, it will modify the quadratic criterion minimized by the adaptation algorithm by introducing a frequency dependent weight.
}

TABLE I

Generalized YK FEedForward COMPENSATORs. Particular CASES

\begin{tabular}{c|c|c}
\hline$A_{Z}, B_{Z}, R_{0}, S_{0}$ & Type of compens. & Ref. \\
\hline \hline$A_{Z}=-1, B_{Z}=0$ & IIR(FIR) & [7], [10], [15] \\
$R_{0}=0, S_{0}=1$ & & {$[17]$} \\
\hline$A_{Z}=A_{M}, B_{Z}=B_{M}$ & YK IIR & {$[16]$} \\
\hline$A_{Z}=A_{M}, B_{Z}=B_{M}$ & YK FIR & $A_{Q}=1$ \\
\hline
\end{tabular}

\section{A. IIR (FIR) adaptive feedforward noise compensators}

For $A_{Z}=-1, B_{Z}=0, R_{0}=0, S_{0}=1$ we are in the context of IIR (FIR) adaptive feedforward compensators discusssed in [15]. The optimal IIR feedforward filter (unknown)taking into account (6) is defined in this case by:

$$
N\left(q^{-1}\right)=\frac{B_{Q}\left(q^{-1}\right)}{A_{Q}\left(q^{-1}\right)},
$$

where $A_{Q}$ and $B_{Q}$ have been defined in (6). The estimated compensator is denoted by $\hat{N}\left(q^{-1}\right)$ or $\hat{N}\left(\hat{\theta}, q^{-1}\right)$ when it is a linear filter with constant coefficients or $\hat{N}\left(t, q^{-1}\right)$ during estimation (adaptation) of its parameters. FIR compensators are obtained by taking $A_{Q}=1$ (i.e. $a_{i}^{Q}=0, \forall i=1: n_{A_{Q}}$ ).

In this context, the filter $\mathrm{L}$ will have the following structures: For FUPLR and NFULMS: $L=\hat{G}$ and

For FUSBA and SFUSBA: $L=\frac{\hat{A}_{M}}{\hat{P}} \hat{G}$, with $\hat{P}=\hat{A}_{M} \hat{S}-$ $\hat{B}_{M} \hat{R}$.

The stability condition associated to the FUPLR is that $\frac{A_{M} G}{P \hat{G}}-\frac{\lambda}{2}=\operatorname{SPR} \quad\left(\lambda=\max \lambda_{2}(t)\right)$ and the stability condition associated with the FUSBA is that: $\frac{A_{M} \hat{P} G}{A_{M} P \hat{G}}-\frac{\lambda}{2}=$ $S P R \quad\left(\lambda=\max \lambda_{2}(t)\right) \cdot{ }^{13}$

The FULMS algorithm will use the same filter as NFULMS but the PAA will be given by (25) instead of (23).

For the case when a negative feedback through $\hat{M}$ is used in order to compensate partially the internal positive feedback (neutralization filter) [25] the same algorithms can be used except that $M$ is replaced by $M^{\prime}=M-\hat{M}$ and $\hat{M}^{\prime}=0$ (with $B_{M}^{\prime}=0$ and $A_{M}^{\prime}=1$ ).

\section{B. IIR-Youla-Kučera parametrized adaptive feedforward compensators}

For $A_{Z}=A_{M}, B_{Z}=B_{M}$ we are in the context of the IIRYK feedforward compensators which has been discussed in $[17]^{14}$. In this context, the filter $L$ will have the structure: For FUPLR and NFULMS: $L=\hat{G}$ and For FUSBA and SFUSBA: $L=\frac{\hat{A}_{M}}{\hat{P}} \hat{G}$, where $\hat{P}=\hat{A}_{Q}\left(A_{M} S_{0}-B_{M} R_{0}\right)$.

The stability conditions are the same as for the IIR configuration but $\hat{P}$ will have a different expression.

\footnotetext{
${ }^{13}$ For the FUSBA algorithm the SPR condition expresses also the allowed tolerance with respect to the uncertainties of the system's models.

${ }^{14}$ This configuration can be interpreted as incorporating a kind of "neutralization filter" [25] (it uses $A_{M}$ and $B_{M}$ ) with the objective to guarantee the stability of the internal positive loop and not for compensating the acoustic feedback.
} 


\section{FIR-Youla-Kučera parametrized adaptive feedforward compensators}

For $A_{Z}=A_{M}, B_{Z}=B_{M}, A_{Q}=1$ we are in the context of the FIRYK feedforward compensator (see [16], [17]). The filters L used in this case are:

For FUPLR and NFULMS: $L=\hat{G}$ and

For FUSBA and SFUSBA: $L=\frac{\hat{A}_{M}}{\hat{P}_{0}} \hat{G}$, where

$\hat{P}_{0}=\left(\hat{A}_{M} S_{0}-\hat{B}_{M} R_{0}\right)$.

The stability condition associated with the FUPLR is that: $\frac{A_{M} G}{P_{0} \hat{G}}-\frac{\lambda}{2}=S P R \quad\left(\lambda=\max \lambda_{2}(t)\right)$. The stability condition associated with the FUSBA is that: $\frac{A_{M} \hat{P_{0}} G}{A_{M} P_{0} \hat{G}}-\frac{\lambda}{2}=$ $S P R \quad\left(\lambda=\max \lambda_{2}(t)\right)$. In this case, for both FUPLR and FUSBA the stability conditions are "global". The main differences with respect to the previous configurations is twofold:

- The FUSBA and SFUSBA algorithms can be implemented from the beginning since $\hat{P}_{0}$ is known and constant and the stability condition is global.

- The design of the central controller can be used to fulfill the SPR conditions for the FUPLR algorithm.

If the central controller is designed such that $P_{0}=A_{M}$ by taking $R_{0}=0, S_{0}=1$, then the algorithms FUPLR and FUSBA are the same and the fulfillment of the SPR condition will depend only on the quality of the estimation of the transfers $\mathrm{G}$ and $\mathrm{M}$. This is a key point because not only the stability of the internal loop will be assured for any finite value of the parameters of the FIR Youla-Kučera filter but in addition the system will be operated under a global stability condition easy to fulfill and allowing to use high values of the adaptation gain leading to fast adaptation.

\section{Design of the Central Controller for YKIIR and YKFIR configurations}

The same central controller $N_{0}\left(q^{-1}\right)=\frac{R_{0}\left(q^{-1}\right)}{S_{0}\left(q^{-1}\right)}$ can be used for FIRYK or IIRYK. The main objective is to guarantee the stability of the internal positive feedback loop for $B_{Q}=$ 0 and $A_{Q}=1$. This can be achieved by using a pole placement design technique (see also [18, Chapter 7]) taking into account that the feedback is positive. All stable poles of the reverse path can be assigned as poles of the closed-loop (one can change their damping). Additional stable poles can be assigned. Sensitivity functions of the internal closed-loop have to be checked.

\section{Youla-Kučera Parametrization-Some Remarks}

Two major observations have to be made when using the Youla-Kučera parametrization:

- If an FIR $Q$ filter is used, the poles of the internal closedloop will be defined by the central controller $R_{0}, S_{0}$ and they will remain unchanged independently of the values of the parameters of the $Q$ filter. The stability condition for the FUSBA algorithm is global.

- If an IIR $Q$ filter is used, the poles of the internal closed-loop will be defined by the central controller but additional poles corresponding to the denominator of the $Q$ filter will be added. The stability condition for the
FUSBA algorithm is local and an initialization with the FUPLR algorithm is necessary.

\section{EXPERIMENTAL RESULTS}

The objective of this section is to asses comparatively the performance of the various adaptive feedforward compensation schemes for attenuating broad-band noise disturbances with unknown and time-varying characteristics.

In defining the experimental protocols, several indicators have to be taken into account and they are discussed below:

\section{Testing Signals}

Two broad-band disturbances have been considered:

- noise with a flat PSD between 70 and $270 \mathrm{~Hz}$

- step change from a flat disturbance $70-170 \mathrm{~Hz}$ to a flat disturbance $170-270 \mathrm{~Hz}$

These disturbances have been obtained using a PRBS with $N=15$ and amplitude 0.1 passed through band-pass Butterworth filters of order 7 with the cut-off frequencies as indicated above.

\section{Number of Adjustable Parameters}

The performances of the various compensators will depend on the number of parameters. An extensive preliminary study has been conducted in order to select a number of parameters offering a compromise between performance in terms of global attenuation and computer load ( [26]). For the IIR and FIR configurations, a number of 30 adjustable parameters have been selected. For the IIRYK and FIRYK configurations, a number of 60 adjustable parameters have been selected.

\section{Type of Parameter Adaptation Algorithms}

For this paper only the adaptive operation will be considered in the experimental evaluation. This means that only the "constant trace adaption gain" and the "constant scalar adaptation gain" have been considered. For a given complexity of the feedforward compensator the performances obtained with various PAA have been evaluated.

\section{Performance measurement}

The attenuation is measured on a sample of $15 \mathrm{~s}$. One expresses in $\mathrm{dB}$ the ratio between the variance of the residual noise in the absence of the compensator and the variance of the residual noise in the presence of the compensator .

\section{Computer Load}

The indicator for the complexity of an algorithm $\triangle T E T$ is evaluated by using the difference between the maximum TET (Task Execution Time) in the presence of the control $\left(T E T_{C L}\right)$ and the minimum TET in open-loop $\left(T E T_{O L}\right)^{15}$. This allows to assess the complexity specifically associated to each algorithm. $\triangle T E T$ is defined as:

$$
\Delta T E T=T E T_{C L}-T E T_{O L}
$$

\footnotetext{
${ }^{15}$ These values are provided by the Matlab RT environment
} 


\section{A. Results for IIR (FIR) Adaptive Feedforward Compensators}

The PAAs used for the experiments are implemented using an initial diagonal gain matrix with a gain of 0.002 per parameter. ${ }^{16}$

Attenuation results for the FIR and IIR configurations are given in rows 1 and 2 of Table II (the first number represents the number of parameters of the numerators and the second number represents the number of parameters of the denominator).

\section{TABLE II}

ATTENUATION FOR VARIOUS CONFIGURATIONS USING THE FUSBA ALGORITHM (70-270 HZ BROAD-BAND DISTURBANCE, $600 \mathrm{~S}$ EXPERIMENTS).

\begin{tabular}{c|c|c|c} 
Filter type & No. params. [num/den] & Attenuation [dB] & $\Delta$ TET [s] \\
\hline FIR & $30 / 0$ & 32.4 & $6.90 \mathrm{e}-5$ \\
\hline IIR & $15 / 15$ & 39.5 & $6.10 \mathrm{e}-5$ \\
\hline IIRYK & $30 / 30$ & 35.7 & $5.67 \mathrm{e}-5$ \\
\hline FIRYK & $60 / 0$ & 28.3 & $5.21 \mathrm{e}-5$
\end{tabular}

TABLE III

EXPERIMENTAL RESULTS FOR IIR 15/15 ADAPTIVE COMPENSATORS USING VARIOUS ADAPTATION ALGORITHMS (70-270 HZ BROAD-BAND DISTURBANCE, $600 \mathrm{~S}$ EXPERIMENTS).

\begin{tabular}{c|c|c} 
Adaptation algorithm & Attenuation [dB] & $\Delta$ TET $[\mathrm{s}]$ \\
\hline Matrix (FUSBA) & 39.5 & $6.10 \mathrm{e}-5$ \\
\hline Matrix (FUPLR) & 35.5 & $4.14 \mathrm{e}-5$ \\
\hline Scalar (SFUSBA) & 36.8 & $5.24 \mathrm{e}-5$ \\
\hline Scalar (NFULMS) & 35.1 & $3.76 \mathrm{e}-5$ \\
\hline Scalar (FULMS) & 34.6 & $3.25 \mathrm{e}-5$
\end{tabular}

Table II indicates that for the same number of parameters the IIR configuration gives a better performance than the FIR configuration. The IIR structure with $15 / 15$ parameters has been chosen to be further evaluated. Results obtained using various adaptation algorithms are shown in Table $\mathrm{III}^{17}$. It can be observed that the matrix gain FUSBA algorithm gives the best results (an improvement of $14.2 \%$ with respect to the performance of the FULMS algorithm). The corresponding scalar version (SFUSBA) shows a loss of $6.8 \%$ in performance and a reduction of the $\triangle T E T$ by $14.1 \%$. The FULMS algorithm gives the lowest performance.

Figure 7 illustrates the evolution of the residual noise and of the attenuation over an horizon of $600 \mathrm{~s}$ for the IIR 15/15 feedforward compensator using the FUSBA algorithm (matrix adaptation gain). Attenuation reaches almost the steady state value at $600 \mathrm{~s}$.

Figure 8 shows the PSD for the FIR 30/0 and the IIR 15/15 using the FUSBA algorithm (matrix adaptation gain). Both compensators assure a significant attenuation of the disturbance. Nevertheless, both PSD show a strong unwanted amplification (around $325 \mathrm{~Hz}$ in the case of the IIR and around $350 \mathrm{~Hz}$ for the FIR) which is caused by the presence of very low damped poles in the internal closed-loop (the

\footnotetext{
${ }^{16}$ This value has been chosen in order to assure a stable operation of the FULMS algorithm.

${ }^{17}$ Experiments on a long horizon of 600 s was necessary in order to get relevant results as a consequence of the slow adaptation
}

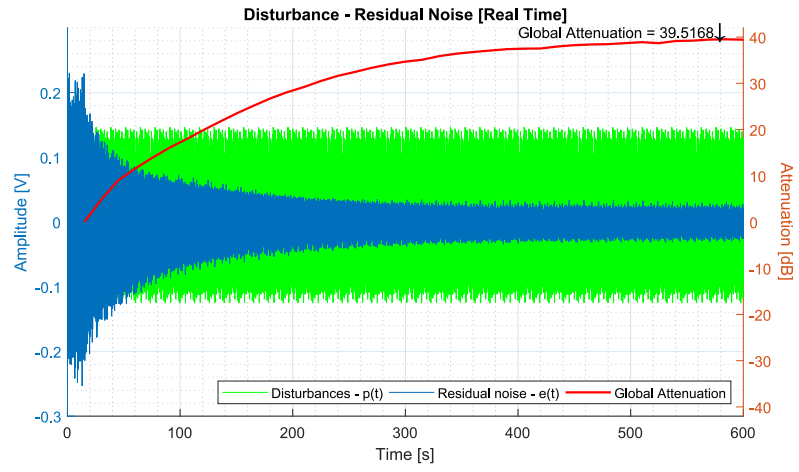

Fig. 7. Residual noise using the IIR $15 / 15$ adaptive compensators using FUSBA matrix adaptation $(70-270 \mathrm{~Hz}$ disturbance, $600 \mathrm{~s}$ experiments).

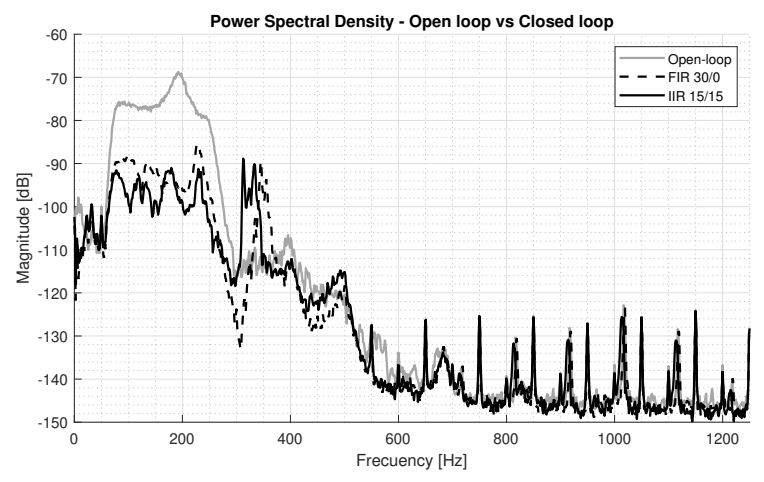

Fig. 8. PSD comparison of FIR $30 / 0$ and IIR 15/15 standard adaptive compensators using FUSBA matrix adaptation $(70-270 \mathrm{~Hz}$ disturbance, $600 \mathrm{~s}$ experiments).

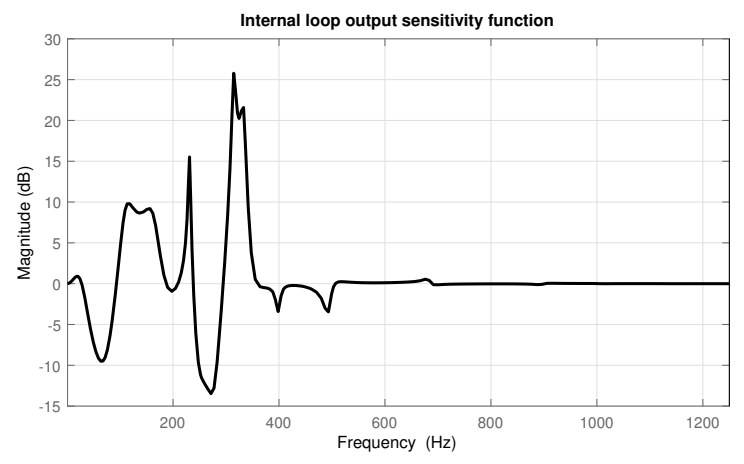

Fig. 9. Estimated internal loop output sensitivity function for the IIR $15 / 15$ adaptive compensator $(70-270 \mathrm{~Hz}$ disturbance, $600 \mathrm{~s}$ experiments).

algorithm guarantees only that the final closed-loop poles will be inside the unit circle but these poles can be very close to the unit circle). Figure 9 shows an estimation of the output sensitivity function of the internal loop (at $600 \mathrm{~s}$ ) for the IIR $15 / 15$ compensator. There is a peak of $25 \mathrm{~dB}$ at $315 \mathrm{~Hz}$ (corresponding to a modulus margin ${ }^{18}$ of 0.06 ) and there is a pair of low damped closed-loop poles at $315 \mathrm{~Hz}$ with a damping of 0.0090. This explains the peak in the PSD of the residual noise. This behavior is a weakness of this approach

\footnotetext{
${ }^{18}$ The modulus margin gives the minimum distance between the Nyquist plot and the critical point $[-1,0]$.
} 
despite excellent attenuation performance.

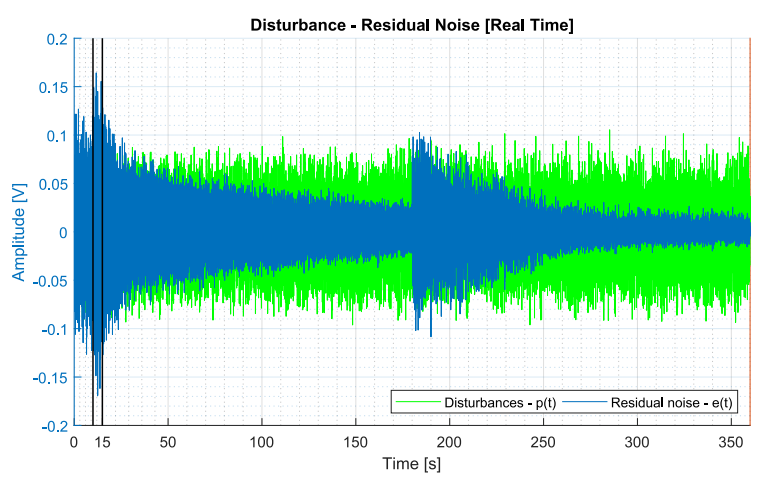

Fig. 10. Residual noise of the IIR $15 / 15$ adaptive feedforward compensator for a change of disturbance from $170-270 \mathrm{~Hz}$ to $70-170 \mathrm{~Hz}$ at $180 \mathrm{~s}$.

Figure 10 illustrates the adaptation capabilities of the IIR 15/15 FUSBA compensator. These experiments are run over $360 \mathrm{~s}$. During the first $15 \mathrm{~s}$, the system is in open-loop and the disturbance $70-170 \mathrm{~Hz}$ is applied until $10 \mathrm{~s}$. Than the disturbance $170-270 \mathrm{~Hz}$ is applied from 10 to $15 \mathrm{~s}$. The adaptive compensation system is in operation from 15 to $360 \mathrm{~s}$. During this period, the disturbance $170-270 \mathrm{~Hz}$ is applied from 15 to $180 \mathrm{~s}$ and the disturbance $70-170 \mathrm{~Hz}$ is applied from 180 to $360 \mathrm{~s}$.

\section{B. Results for IIRYK Adaptive Feedforward Compensators}

The initial diagonal adaptation gain matrix used for IIRYK compensators has been set at 0.02 per parameter and the same value has been used for the constant scalar adaptation gain.

Row 3 in Table II shows the attenuation that can be obtained with the IIRYK feeforward compensator. Table IV gives

\section{TABLE IV}

EXPERIMENTAL RESULTS FOR IIRYK 30/30 ADAPTIVE COMPENSATORS USING VARIOUS ADAPTATION ALGORITHMS (70-270 HZ BROAD-BAND DISTURBANCE, $180 \mathrm{~S}$ EXPERIMENTS).

\begin{tabular}{c|c|c} 
Adaptation algorithm & Attenuation [dB] & $\Delta$ TET [s] \\
\hline Matrix (FUSBA) & 30.2 & $5.59 \mathrm{e}-5$ \\
\hline Matrix (FUPLR) & 6.1 & $5.48 \mathrm{e}-5$ \\
\hline Scalar (SFUSBA) & 27.5 & $4.21 \mathrm{e}-5$ \\
\hline Scalar (SFUPLR) & 6.1 & $3.87 \mathrm{e}-5$
\end{tabular}

a comparison of the various adaptation algorithms in terms of global attenuation and $\triangle T E T$. Clearly the FUSBA and the SFUSBA give the best results. The loss in performance when using a scalar adaptation gain is around $9 \%$ and the corresponding reduction of the $\triangle T E T$ is about $24 \%$. To understand why the FUPLR gives in this case far less good results than does the FUSBA, one has to look at the phase of the estimated $\frac{A_{M}}{P}$ shown in Figure 11. One can see that $\frac{A_{M}}{P}$ is not positive real between $50-120 \mathrm{~Hz}, 160-310 \mathrm{~Hz}$ and $350-890 \mathrm{~Hz}$. It is clear that in a large frequency spectrum the adaptation will not move in the right direction even if averaging arguments can be taken into account.
Figure 12 illustrates the evolution of the residual noise and of the attenuation over an horizon of $600 \mathrm{~s}$ for the IIRYK 15/15 feedforward compensator using the FUSBA algorithm (matrix adaptation gain). Attenuation reaches almost the steady state value at $600 \mathrm{~s}$ and is faster than for the IIR using the same adaptation gain.

Figure 13 shows the PSD for the IIRYK 30/30 using the FUSBA algorithm. The peak at $306 \mathrm{~Hz}$ is due to a low damped pair of complex poles of the internal loop. The peak at $306 \mathrm{~Hz}$ is much lower in this case than the peak observed in the case of IIR compensator in the same frequency region (see Figure 8). It can be concluded that the closed-loop poles in this region are more damped and that the stability margin is improved.

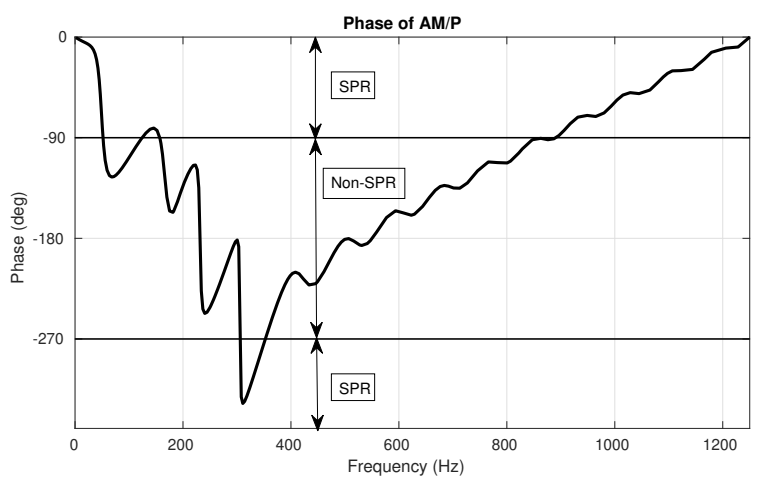

Fig. 11. Phase of the estimated $\frac{A_{M}}{P}$ for the IIRYK 30/30 adaptive compensator (70-270 Hz disturbance, $600 \mathrm{~s}$ experiments).

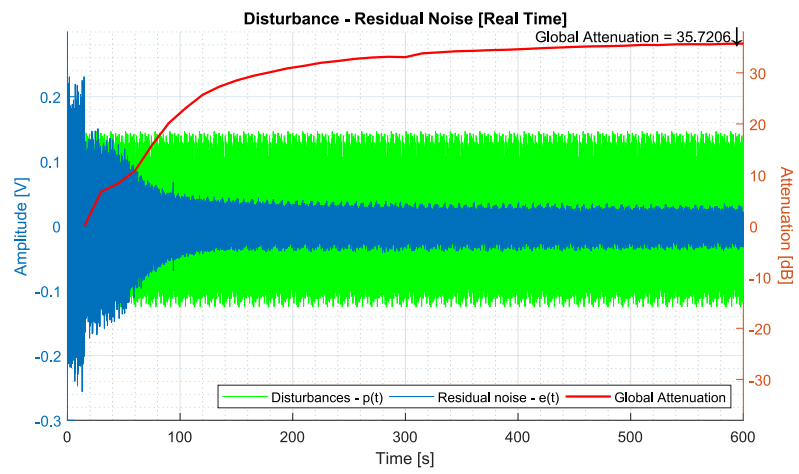

Fig. 12. Residual noise using the IIRYK $30 / 30$ adaptive compensators with FUSBA matrix adaptation $(70-270 \mathrm{~Hz}$ disturbance, $600 \mathrm{~s}$ experiments).

Figure 14 shows the adaptation capabilities of the IIRYK adaptive compensator with 30/30 parameters. The same protocol is used as in the case of the standard IIR. The transients are shorter than for the IIR 15/15 while the steady states are comparable (even if one is better in high frequencies (IIRYK) and the other one is better in lower frequencies (IIR)).

\section{Results for FIRYK Adaptive Feedforward Compensators}

The initial diagonal adaptation gain matrix used for FIRYK compensators has been set at 0.5 per parameter and the same value has been used for the constant scalar adaptation gain 


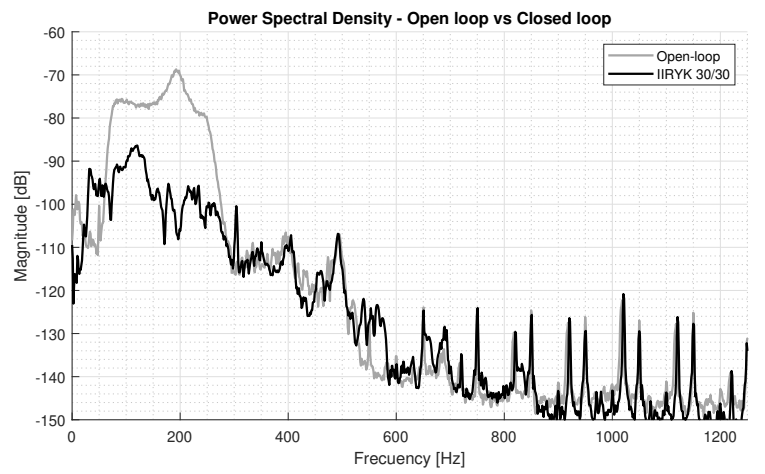

Fig. 13. PSD of the IIRYK $30 / 30$ adaptive compensators using FUSBA matrix adaptation $(70-270 \mathrm{~Hz}$ disturbance, $600 \mathrm{~s}$ experiments).

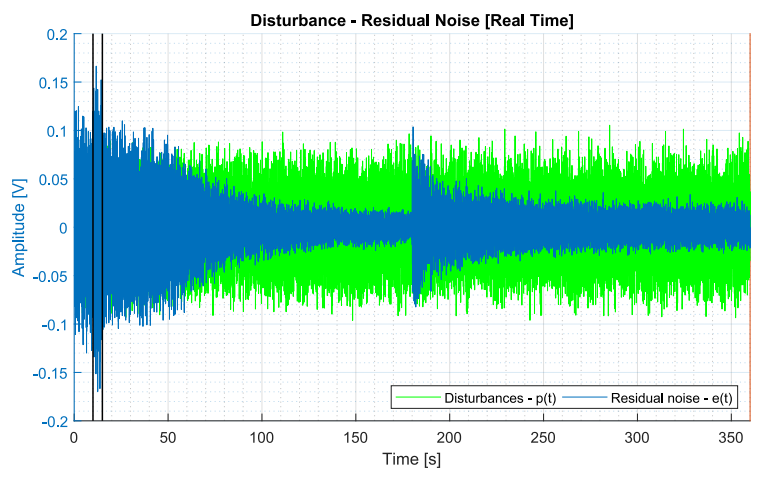

Fig. 14. Residual noise of the IIRYK 30/30 adaptive feedforward compensator for a change of disturbance from $170-270 \mathrm{~Hz}$ to $70-$ $170 \mathrm{~Hz}$ at $180 \mathrm{~s}$.

(this high value of the adaptation gain can be used since one can take advantage of the global character of the stability condition for the FUSBA and SFUSBA algorithms in this case).

The attenuation obtained with the FIRYK configuration is shown in the row 4 of Table II. For the same complexity the IIRYK offers better performance than the FIRYK.

TABLE V

EXPERIMENTAL RESULTS FOR FIRYK 60/0 ADAPTIVE COMPENSATORS USING VARIOUS ADAPTATION ALGORITHMS (70-270 HZ BROAD-BAND DISTURBANCE, $180 \mathrm{~S}$ EXPERIMENTS).

\begin{tabular}{c|c|c} 
Adaptation algorithm & Attenuation [dB] & $\Delta$ TET $[\mathrm{s}]$ \\
\hline Matrix (FUSBA) & 27.0 & $5.10 \mathrm{e}-5$ \\
\hline Matrix (FUPLR) & unstable & - \\
\hline Scalar (SFUSBA) & 26.7 & $3.94 \mathrm{e}-5$ \\
\hline Scalar (SFUPLR) & unstable & -
\end{tabular}

Table $\mathrm{V}$ gives a comparison of the various adaptation algorithms in terms of global attenuation and $\triangle T E T$. It was observed that the FUPLR is unstable and this can be understood when looking to the phase plot of the estimated $\frac{A_{M}}{P}$ given in Figure 15. $\frac{A_{M}}{P}$ is not positive real in a large frequency range from $110 \mathrm{~Hz}$ to $760 \mathrm{~Hz}$ and therefore one absolutely needs to use the FUSBA algorithm which for the this configuration provide a global stability and does not requires initialization. ${ }^{19}$ The loss in performance when using a scalar adaptation gain is very small in this case (1\%) while the computer load decreases by $23 \%$.

Figure 16 shows both the time evolution of the residual error and of the attenuation. While the adaptation is much faster compared with the previous schemes the steady state is less good (28.3 dB compared with the $35.7 \mathrm{~dB}$ for the IIRYK $30 / 30$ and the $39.5 \mathrm{~dB}$ for the IIR $15 / 15$ and the $32.4 \mathrm{~dB}$ for the FIR 30/0).

Figure 17 shows the PSD of the FIRYK 60/0 using the FUSBA algorithm. The loss in performance with respect to the other schemes seems to occur in the region $210-270 \mathrm{~Hz}$. Nevertheless, this curve indicates that the peak around $325 \mathrm{~Hz}$ is lower than in the previous cases (it will depend in fact on the design of the central controller).

Figure 18 shows the estimation of the output sensitivity function of the internal loop for the FIRYK 60/0 using the FUSBA algorithm. As one can see, the maximum is about $10 \mathrm{~dB}$ which assures from the beginning of the adaptation process a modulus margin greater than 0.3 .

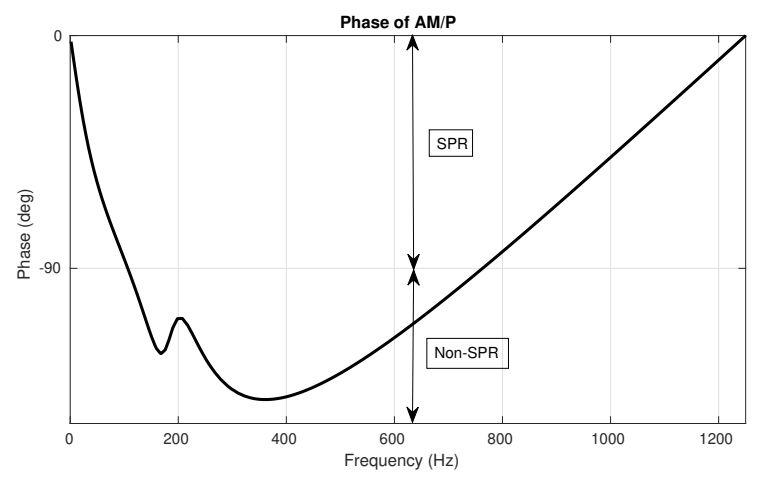

Fig. 15. Phase of estimated $\frac{\boldsymbol{A}_{M}}{\boldsymbol{P}}$ for the FIRYK $60 / 0$ adaptive compensator $(70-270 \mathrm{~Hz}$ disturbance, $600 \mathrm{~s}$ experiments).

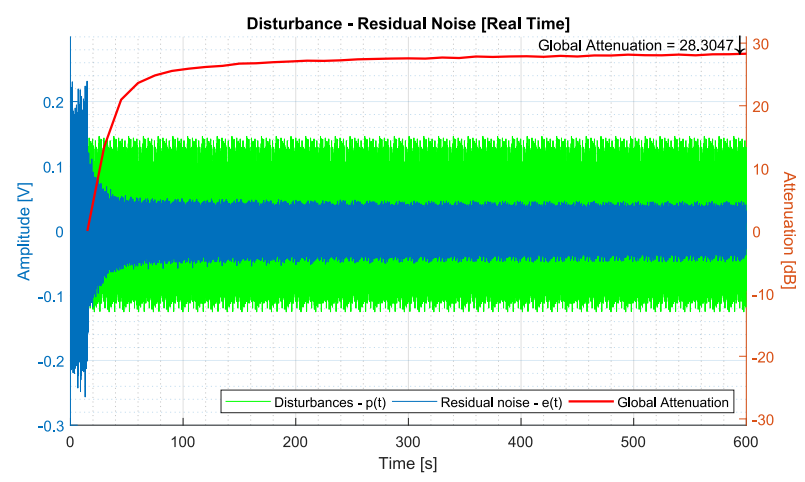

Fig. 16. Residual noise using the FIRYK 60/0 adaptive compensators with FUSBA matrix adaptation $(70-270 \mathrm{~Hz}$ disturbance, $600 \mathrm{~s}$ experiments).

Figure 19 shows the adaptation capabilities of the FIRYK adaptive compensator with $60 / 0$ parameters. The same protocol is used as in the case of the standard IIR. As expected, the

\footnotetext{
${ }^{19}$ Reducing drastically the adaptation gain, FUPLR may work but the performance will be highly degraded.
} 


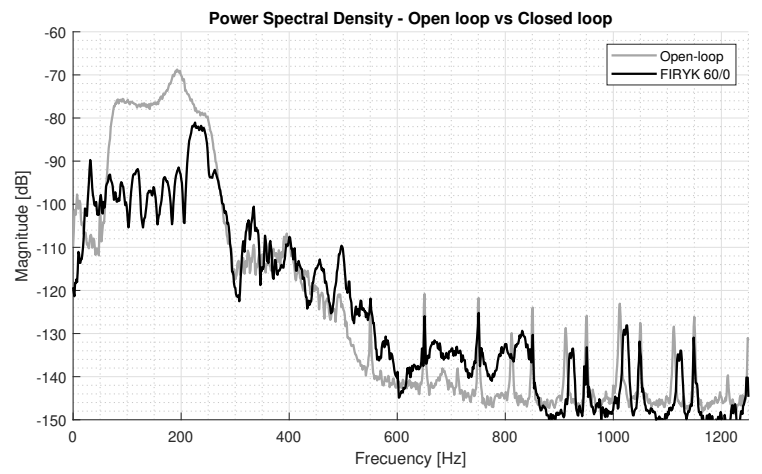

Fig. 17. PSD of the FIRYK 60/0 adaptive compensators using FUSBA matrix adaptation (70-270 $\mathrm{Hz}$ disturbance, $600 \mathrm{~s}$ experiments).

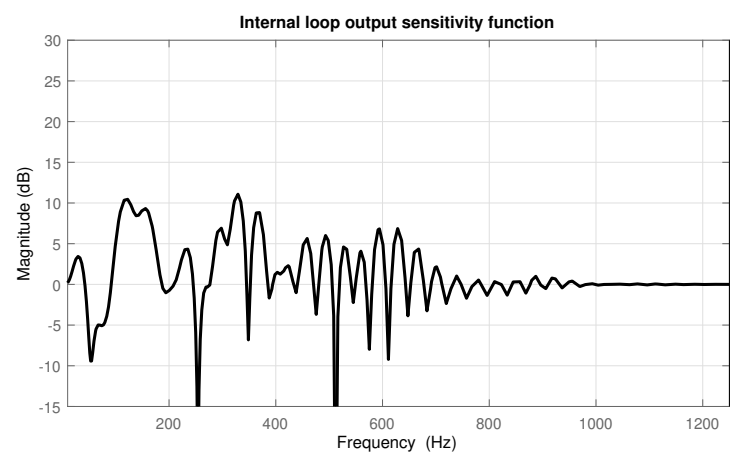

Fig. 18. Internal loop output sensitivity function for the FIRYK 60/0 adaptive compensator (70-270 Hz disturbance, $600 \mathrm{~s}$ experiments).

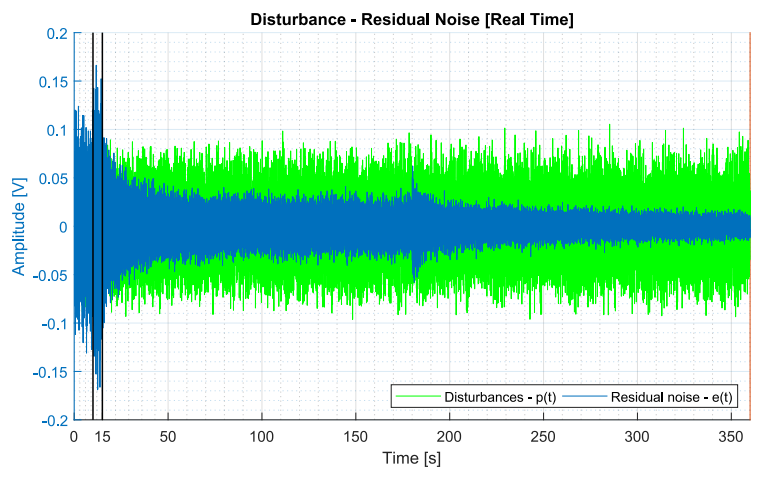

Fig. 19. Residual noise of the FIRYK 60/0 adaptive feedforward compensator for a change of disturbance from $170-270 \mathrm{~Hz}$ to 70 $170 \mathrm{~Hz}$ at $180 \mathrm{~s}$.

adaption transient is very fast and in addition the maximum value of the residual noise during the adaptation transient is much smaller compared with the IIR and the IIRYK.

\section{Filtering the Residual Noise for Parameter Adaptation}

Figure 20 illustrates the effect of using a filtered residual noise in the adaptation algorithm upon the PSD of the residual noise. The comparison is done using the IIRYK 30/30 compensator with the FUSBA algorithm over $180 \mathrm{~s}$ (similar behavior is obtained also for the other compensator structures). The residual noise filter considered is a low pass FIR filter given

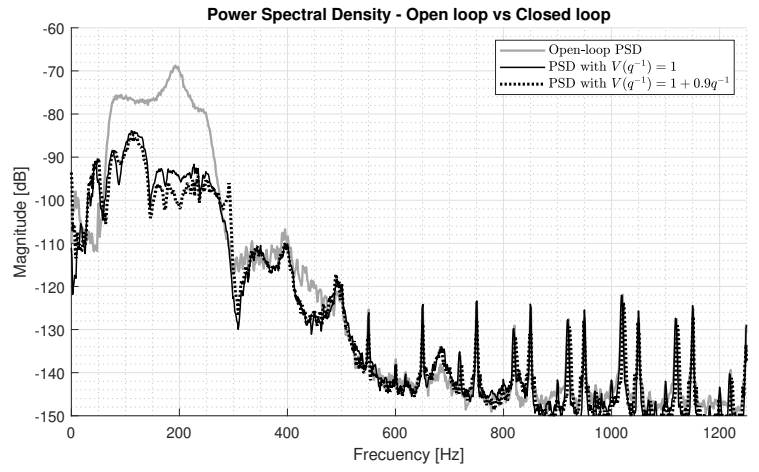

Fig. 20. PSD comparison of the residual noise, using the adaptive compensator without filtering of the residual noise (solid black line), and using the adaptive compensator with filtering of the residual noise (dotted black line).

by

$$
V\left(q^{-1}\right)=1+0.9 q^{-1}
$$

The frequency response of this filter is shown in Fig. 21. As it can be observed, this filter enhances the attenuation of disturbances at low frequencies. The global attenuation obtained with this filter is $32.9 \mathrm{~dB}$ while without this filter it is $30.2 \mathrm{~dB}$ (a performance improvement of about $9 \%$ ).

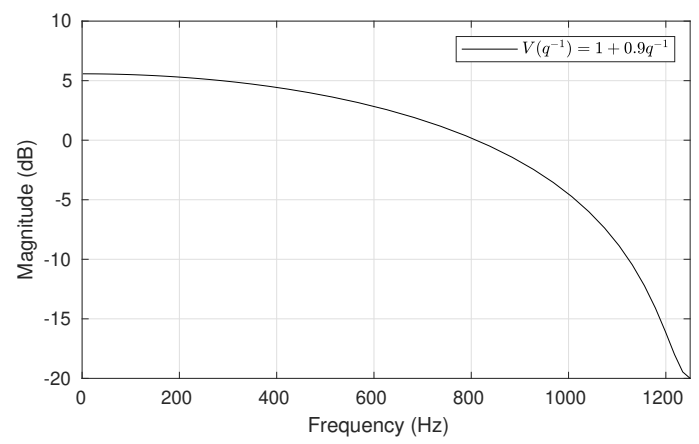

Fig. 21. Residual error filter.

\section{Performance comparison - a summary}

For a comparable complexity in terms of computer load, the IIR feeforward compensator with the matrix adaptation gain using the FUSBA algorithm provides the best steady state results in terms of attenuation followed by the IIRYK, FIR and FIRYK. In terms of adaptation transients, the FIRYK provides the best results. In terms of safety of operation (stability of the internal positive loop) without any doubt the FIRYK using the FUSBA algorithm (matrix adaptation gain) is the good choice since in this case the stability of the internal positive loop depends exclusively upon the stabilizing central controller. The steady state performance of the FIRYK can be enhanced by augmenting the number of adjustable parameters (which will increase however the computer load).

The use of the scalar adaptation gain leads to a degradation of the performances and to a reduction of the computer load (except to some extent for the FIRYK - see Table V). 
For IIR compensators FUSBA or SFUSBA algorithms provide better results than the now classical FULMS algorithm.

The use of a filtered residual noise measurement for adaptation may improve the overall performance.

\section{CONCLUSION}

This study has provided the opportunity to assess comparatively the properties of various structures and algorithms which can be used for adaptive feedforward noise compensation taking into account the inherent presence of an internal positive coupling in most of the applications. In many practical applications instabilities have been encountered using classical algorithms (FXLMS, FULMS, etc.) which do not take into account this internal positive coupling. Furthermore this study has been conducted on an experimental configuration far from the almost co-location of the compensator loudspeaker and of the residual noise measurement currently used in the litterature of ANC.

Based on extensive experimental tests one can state that FIRYK adaptive compensator structure using the FUSBA (or SFUSBA) algorithm is the good solution for a robust operation of the feedforward active noise attenuation. The main argument is that the stability of the internal positive loop is guaranteed by the design of the central controller and is not influenced by the evolution of the adjustable filter parameters.

\section{APPENDIX}

\section{IDENTIFICATION OF THE EXPERIMENTAL TEST-BENCH}

The identification procedure follows closely the procedure described in [27], [28] The PRBS characteristics used in the identification process as excitation signal was: magnitude $=$ $0.14 \mathrm{~V}$, register length $=15$, frequency divider of 1 , sequence length: $2^{15}-1=32767$ samples, guaranteeing a uniform power spectrum up to $1250 \mathrm{~Hz}$.

Once the input-output data have been acquired, the next step in the identification procedure is to estimate the order $n=\max \left(n_{A}, n_{B}+d\right)$ of the model from experimental data. The method of Duong ( [18], [29]) has been used. In the method of Duong, the minimum of a quadratic criterion in terms of an unbiased plant-model error penalized by a complexity terms is searched. But since the minimum was relatively flat, nearby values have also been considered. The final selection has been done by checking what order allows to capture all the oscillatory modes in the model and lead to the best statistical validation once the parameters are identified.

Comparative parameter estimations considering various plant + noise models and estimation algorithms led to the conclusion that an ARMAX model representation is the most appropriate for this system and the best results in terms of statistical validation (whiteness test on the residual error) have been obtained using the Output Error with Extended Prediction Model (termed OEEPM or XOLOE) [21]. The orders of the identified models are given in Table VI.

\section{REFERENCES}

[1] S. Elliott and P. Nelson, "Active noise control," Noise / News International, pp. 75-98, June 1994.
TABLE VI

ORDERS OF THE IDENTIFIED SYSTEM PATHS.

\begin{tabular}{c|c|c|c}
\hline Model & $n_{A}$ & $n_{B}$ & $d$ \\
\hline \hline Primary(global) & 24 & 20 & 7 \\
\hline Secondary & 26 & 27 & 6 \\
\hline Reverse & 25 & 22 & 5 \\
\hline
\end{tabular}

[2] S. Elliott and T. Sutton, "Performance of feedforward and feedback systems for active control," Speech and Audio Processing, IEEE Transactions on, vol. 4, no. 3, pp. $214-223$, may 1996.

[3] S. Kuo and D. Morgan, "Active noise control: a tutorial review," Proceedings of the IEEE, vol. 87, no. 6, pp. 943 - 973, jun 1999.

[4] J. Zeng and R. de Callafon, "Recursive filter estimation for feedforward noise cancellation with acoustic coupling," Journal of Sound and Vibration, vol. 291, no. 3-5, pp. 1061 - 1079, 2006.

[5] B. Widrow and S. Stearns, Adaptive Signal Processing. Englewood Cliffs, New Jersey: Prentice-Hall, 1985.

[6] M. Kuo and D. Morgan, Active noise control systems-Algorithms and DSP implementation. New York,: Wiley, 1996.

[7] C. Jacobson, J. Johnson, C.R., D. McCormick, and W. Sethares, "Stability of active noise control algorithms," Signal Processing Letters, IEEE, vol. 8, no. 3, pp. $74-76$, mar 2001.

[8] F. Ben Amara, P. Kabamba, and A. Ulsoy, "Adaptive sinusoidal disturbance rejection in linear discrete-time systems - Part I: Theory," Journal of Dynamic Systems Measurement and Control, vol. 121, pp. 648-654, 1999.

[9] —-, "Adaptive sinusoidal disturbance rejection in linear discretetime systems - Part II: Experiments," Journal of Dynamic Systems Measurement and Control, vol. 121, pp. 655-659, 1999.

[10] L. Eriksson, "Development of the filtered-U LMS algorithm for active noise control," J. of Acoustical Society of America, vol. 89, no. 1, pp. 257-261, 1991.

[11] A. Wang and W. Ren, "Convergence analysis of the Filtered-U algorithm for active noise control," Signal Processing, vol. 83, pp. 1239-1254, 1999.

[12] R. Fraanje, M. Verhaegen, and N. Doelman, "Convergence analysis of the Filtered-U LMS algorithm for active noise control in case perfect cancellation is not possible," Signal Processing, vol. 73, pp. 255-266, 2003.

[13] L. Xie, Z. cheng Qiu, and X. min Zhang, "Vibration control of a flexible clamped-clamped plate based on an improved FULMS algorithm and laser displacement measurement," Mechanical Systems and Signal Processing, vol. 75, pp. 209 - 227, 2016.

[14] X. Zhu, Z. Gao, Q. Huang, S. Gao, and E. Jiang, "Analysis and implementation of MIMO FULMS algorithm for active vibration control," Transactions of the Institute of Measurement and Control, vol. 34, no. 7, pp. 815-828, 2012.

[15] I. D. Landau, M. Alma, and T.-B. Airimitoaie, "Adaptive feedforward compensation algorithms for active vibration control with mechanical coupling," Automatica, vol. 47, no. 10, pp. 2185 - 2196, 2011.

[16] I. D. Landau, T.-B. Airimitoaie, and M. Alma, "A Youla-Kučera parametrized adaptive feedforward compensator for active vibration control with mechanical coupling," Automatica, vol. 48, no. 9, pp. 2152 $-2158,2012$.

[17] _ , "IIR Youla-Kučera parameterized adaptive feedforward compensators for active vibration control with mechanical coupling," IEEE Transactions on Control System Technology, vol. 21, no. 3, pp. 765779, May 2013.

[18] I. D. Landau, T.-B. Airimitoaie, A. Castellanos Silva, and A. Constantinescu, Adaptive and Robust Active Vibration Control-Methodology and Tests, ser. Advances in Industrial Control. Springer Verlag, 2017.

[19] R. Fraanje, "Robust and fast schemes in broadband active noise and vibration control," Ph.D. dissertation, University of Twente, Twente, The Netherlands, 2004.

[20] J. Hu and J.-F. Lin, "Feedforward active noise controller design in ducts without independent noise source measurements," IEEE Trans. on Control System Technology, vol. 8, no. 3, pp. 443-455, 2000.

[21] I. D. Landau, R. Lozano, M. M'Saad, and A. Karimi, Adaptive control, 2nd ed. London: Springer, 2011.

[22] B. Anderson, R. Bitmead, C. Johnson, P. Kokotovic, R. Kosut, I. Mareels, L. Praly, and B. Riedle, Stability of adaptive systems. Cambridge Massachusetts , London, England: The M.I.T Press, 1986. 
[23] L. Ljung and T. Söderström, Theory and practice of recursive identification. Cambridge Massachusetts, London, England: The M.I.T Press, 1983.

[24] J. Treichler, M. Larimore, and J. Johnson, C., "Simple adaptive IIR filtering," in Acoustics, Speech, and Signal Processing, IEEE International Conference on ICASSP '78., vol. 3, apr 1978, pp. 118 - 122.

[25] M. Tufaila, S. Ahmeda, M. Rehana, and M. T. Akhtarb, "A two adaptive filters-based method for reducing effects of acoustic feedback in singlechannel feedforward anc systems," Digital Signal Processing, vol. 90, pp. 18-27, 2019.

[26] R. Melendez, "Robust and adaptive feedback noise attenuation in ducts," Ph.D. dissertation, University of Grenoble, France, May 2019.

[27] R. Melendez, I. Landau, L. Dugard, and G. Buche, "Data driven design of tonal noise feedback cancellers," in Proceedings of the 20th IFAC World Congress, Toulouse, France, 2017, pp. 916-921.

[28] I. D. Landau, R. Melendez, L. Dugard, and G. Buche, "Robust and adaptive feedback noise attenuation in ducts," IEEE Transactions on Control System Technology, vol. 27, no. 2, pp. 872-879, March 2019.

[29] H. N. Duong and I. D. Landau, "An IV based criterion for model order selection," Automatica, vol. 32, no. 6, pp. 909-914, 1996. 